\title{
ESCAPE DE AIRE NASAL DE FONEMAS OCLUSIVOS Y FONEMAS FRICATIVOS EN PACIENTES PORTADORES DE FISURA LABIOVELOPALATINA UNILATERAL OPERADA
}

\author{
NASAL AIR ESCAPE DURING THE EMISSION OF PLOSIVE AND \\ FRICATIVE PHONEMES IN PATIENTS WITH \\ OPERATED UNILATERAL CLEFT LIP AND PALATE
}

\author{
María Angélica Fernández ${ }^{1}$ \\ Braulio Gómez ${ }^{2}$ \\ ${ }^{1}$ Fonoaudióloga, Escuela de Fonoaudiología, Facultad de Medicina, Universidad de Chile. \\ ${ }^{2}$ Fonoaudiólogo, Departamento del Niño y Ortopedia Dento Maxilar, \\ Facultad de Odontología, Universidad de Chile. \\ Correspondencia a: ma_fernandez@med.uchile.cl
}

\begin{abstract}
RESUMEN
Objetivo: determinar si existen diferencias en el escape de aire nasal durante la emisión de fonemas oclusivos y fonemas fricativos en pacientes portadores de fisura labiovelopalatina unilateral operada. Material y método: Para la presente investigación se utilizó un instrumento llamado Aerofonoscopio llc que capta el escape de aire nasal de los pacientes al hablar. El estudio fue realizado en 74 pacientes diagnosticados con fisura labiovelopalatina unilateral operada, con un rango de edad desde los 58 meses (4 años 10 meses) hasta los 540 meses (45 años). Para realizar el análisis comparativo se utilizó la prueba "t" student. Resultados: Se encontraron diferencias estadísticamente significativas en el porcentaje de escape de aire nasal entre fonemas fricativos $(52,22 \%$ ) y fonemas oclusivos (35,94\%). Conclusión: Los fonemas fricativos son los más afectados en portadores de fisura labiovelopalatina. El fonema fricativo más alterado es el fonema /s/. La aerofonoscopía es un examen objetivamente válido para determinar el grado de escape nasal.
\end{abstract}

Palabras claves: Aerofonoscopía, fonemas oclusivos, fonemas fricativos, fisura labiovelopalatina.

\begin{abstract}
Objective. The objective of the present study is to determine whether or not differences in the nasal air escape during the emission of plosive and fricative phonemes in patients with unilateral cleft lip and palate exist. Methods. An aerophonoscope Ilc, an instrument which captures the nasal air escape during speech, was used. The study sample comprised 74 patients diagnosed with operated unilateral cleft lip and palate. The age of the participants ranged between 58 ( 4 years and 10 months) and 540 months ( 45 years). A t-test for group comparisons was computed for the statistical analysis. Results. Statistically significant differences were found in the percentage of nasal air escape between fricative $(52,22 \% 9)$ and plosive phonemes $(35,94 \%)$. Conclusion. Fricative phonemes are the most affected ones among patients with unilateral cleft lip and palate. The most affected fricative phoneme is $/ \mathrm{s} /$. The aerophonoscopy is an objective and valid test to determine the nasal air escape.
\end{abstract}

Key words: Aerophonoscopy, plosive phoneme, fricative phoneme, lip and cleft palate. 


\section{INTRODUCCIÓN}

En pacientes portadores de fisuras labiovelopalatinas es frecuente encontrar alteraciones funcionales una vez que han sido operados, una de éstas es la función del velo del paladar. La acción del velo del paladar permite diferenciar los fonemas orales de los fonemas que son nasales ${ }^{(1)}$. Durante la función de fonoarticulación, el esfínter velofaríngeo obstruye el paso de energía sonora hacia la cavidad nasal cuando las emisiones son orales y permite dicho paso de energía cuando las emisiones son nasales.

Cuando este esfínter no está funcionando eficientemente, permite el paso de energía sonora hacia la cavidad nasal durante la producción de emisiones orales, lo que se denomina "hipernasalidad". Si por el contrario, el paso de energía sonora hacia la cavidad nasal durante la emisión de los fonemas nasales es obstruido a nivel nasal, por ejemplo, por desviación del tabique o cornetes hiperplásicos, se producirá la llamada "hiponasalidad". Cuando están ambos tipos de fonemas afectados, se tendrá una "Nasalidad Mixta"(2-4).

Una de las evaluaciones que se realizan para poder determinar la funcionalidad y competencia del esfínter velofaríngeo es la medición del grado de nasalidad que el niño presenta al momento de emitir distintos fonemas ${ }^{(5)}$. Sin embargo, cuantificar y objetivar la nasalización es uno de los mayores inconvenientes en el diagnóstico de las cualidades de la voz, ya que la nasalidad es el síntoma patognomónico en estas malformaciones ${ }^{(5-8)}$.

Existen distintas alternativas de evaluación para poder determinar el grado de nasalidad de los fonemas orales, entre las que se encuentran las descritas por Henningsson ${ }^{(5)}$, LohmanderAgerskov ${ }^{(9)}$, Vallino-Napoli(10), Sánchez ${ }^{(11)}$ y Cortés ${ }^{(12)}$.

- Espejo de Glatzel, que permite visualizar el escape por vía nasal al momento de emitir sonidos, sílabas o palabras.

- Radiografía lateral de velo del paladar.

- Nasofaringoscopía permite evaluar los movimientos del velo del paladar y de las paredes laterales de la faringe mientras emite ciertos fonemas.

- Videofluoroscopía ayudará a diagnosticar insuficiencia velofaríngea. 
Sin embargo, todos los exámenes mencionados anteriormente no permiten evaluar objetivamente el escape de aire nasal en los fonemas evaluados, ya que dependen de la experiencia del evaluador para poder determinar el grado de escape nasal, dichos exámenes son imprecisos e incompletos. Por lo anterior, es necesario contar con un método de diagnóstico gráfico, completo y confortable para el paciente.

Durante los últimos años se ha estado utilizando un método de evaluación llamado aerofonoscopía $^{(13-15)}$. Este examen cuenta con un instrumento, el aerofonoscopio Ilc, que capta el escape de aire nasal de los pacientes al hablar y lo registra en una pantalla de cristal líquido. El aerofonoscopio llc cuenta con una unidad central que es un computador y una sonda constituida por un micrófono unidireccional pequeño, además de dos orificios en su cara superior que se corresponden con las narinas de las fosas nasales de los pacientes.

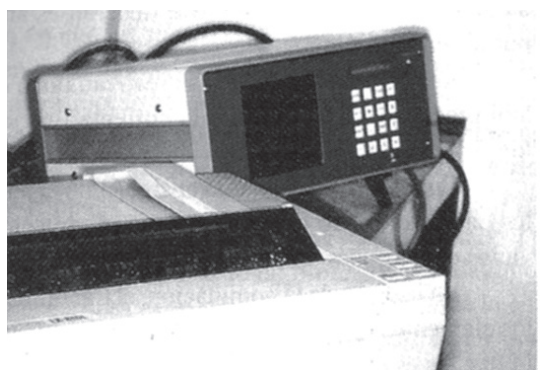

b)

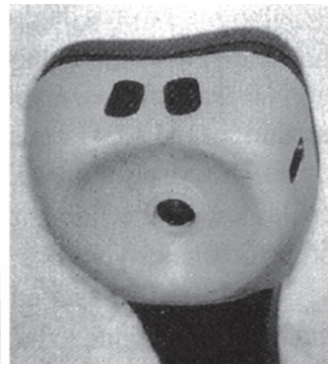

Figura 1: Aerofonoscopio Ilc; a) Unidad Central; b) Sonda

Las investigaciones internacionales realizadas con exámenes convencionales, señalan que los fonemas más alterados en los pacientes fisurados de paladar y velo son los fonemas oclusivos, que se ven más afectados por la baja presión intraoral y en menor grado los fonemas fricativos $^{(5,7-9,16)}$.

Existen algunas investigaciones realizadas con aerofonoscopía que sugieren que los fonemas más afectados en pacientes portadores de fisuras labiovelopalatinas, serían los fonemas fricativos ${ }^{(3,13,15)}$.

El objetivo del presente trabajo es determinar si existen diferencias en el escape de aire nasal durante la emisión de fonemas oclusivos y fonemas fricativos en pacientes portadores de fisura labiovelopalatina unilateral operada. 


\section{MATERIAL Y MÉTODO}

Para la presente investigación se utilizó un instrumento llamado Aerofonoscopio IIc ${ }^{(13-15)}$, instrumento de origen francés, que cuenta con los siguientes elementos:

1. Unidad Central con un computador, que mediante un teclado digital, permite seleccionar el examen que se va a practicar y una pantalla de cristal líquido dividida horizontalmente en dos mitades.

2. Una sonda, conectada a la Unidad Central, que posee:

- Un micrófono unidireccional en la cara anterior que es cóncava.

- Dos orificios que se corresponden con las narinas en la cara superior de la sonda, para captar los escapes de aire.

- Filamentos ultra sensibles a alta temperatura, que capturan las variaciones de escape de aire.

- Dos orificios en las caras laterales para permitir la normal ventilación del paciente.

- Una impresora de cinta conectada a la unidad central, que permite dejar la impresión de todo el test que se utilice, 0 ,

- Un computador con hardware y software que reconozcan al Aerofonoscopio donde se puede almacenar la información paciente a paciente con los exámenes que se le practiquen.

El operador utiliza gorro, mascarilla y guantes para practicar el examen.

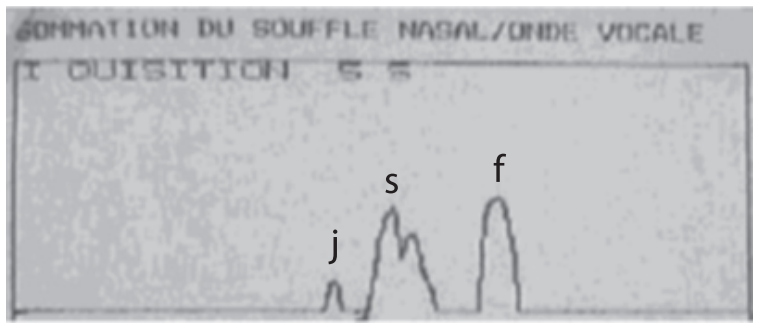

Figura 2. Ejemplo de gráfico en la pantalla del aerofonoscopio llc durante la emisión de los fonemas /s/, /fl, /j/ 
Los exámenes que puede realizar el aerofonoscopio son los siguientes:

- Aerofonoscopía a pacientes portadores de alguna noxa de velo del paladar.

- $\quad$ Escape de aire y energía sonora por fosa nasal izquierda.

- $\quad$ Escape de aire y energía sonora por fosa nasal derecha.

- Suma de escape de aire nasal.

- Inspiración por fosa nasal derecha.

- Inspiración por fosa nasal izquierda.

- $\quad$ Suma de inspiración por fosa nasal derecha e izquierda.

La aerofonoscopía cuenta con un protocolo de examen que se practica en la Facultad de Odontología de la Universidad de Chile, y que permite analizar los resultados con los mismos parámetros. El protocolo es el siguiente:

1. Repetir la fórmula fonética con fonemas oclusivos TA PI KE

2. Repetir la fórmula fonética con fonemas nasales NO MA MA (del test fonético francés original, el cual no fue modificado).

3. Repetir la fórmula fonética con fonemas fricativos y africados SE FI CHU

4. Repetir la fórmula fonética con fonemas orales BA LA DA

5. Repetir la fórmula fonética con fonemas fricativos SE FI JU

6. Repetir la fórmula fonética con fonemas africados CHI LLA

7. Ventilación por fosa nasal derecha e izquierda.

8. Suma de aire nasal

El estudio fue realizado en 74 pacientes diagnosticados con fisura labiovelopalatina unilateral operada. A cada uno de estos pacientes se le realizó una aerofonoscopía. Todos los exámenes aerofonoscópicos fueron realizados por un solo evaluador, el que aplicaba la sonda bajo las narinas del paciente mientras éste va repitiendo cada fórmula fonética. Los exámenes aerofonoscópicos fueron realizados desde 1996 al 2005 y se cuenta con los archivos respectivos. 
El rango de edad de los 74 pacientes evaluados fluctuaba entre los 58 meses (4 años 10 meses) hasta los 540 meses (45 años).

La muestra está compuesta por 37 pacientes de género femenino y 37 pacientes de género masculino. De toda la muestra, 28 pacientes presentaban fisura labiovelopalatina derecha operada y 46 pacientes presentaban fisura labiovelopalatina izquierda operada.

A pesar que se utilizó todo el protocolo, para efectos de este estudio se ocupó y analizó sólo los fonemas oclusivos /t/, /p/ y /k/ y los fonemas fricativos / s/, /f/ y /j/.

Para determinar el grado de escape de aire nasal fue necesario establecer una fórmula para poder porcentualizar el escape de aire en cada fórmula fonética evaluada. Se utilizó la propuesta por Bollo(13) y Gómez ${ }^{(15)}$. Se ocupó el gráfico que dibuja el computador en la pantalla al registrarse algún escape de aire nasal durante la emisión de las respectivas fórmulas fonéticas. Este gráfico describe una curva en la zona superior de la pantalla, de esta manera, la curva graficada fue considerada para la medición estadística como un triángulo, entonces se registró el área de escape nasal de cada fonema calculando el área de dicho triángulo.

\section{Área Triángulo $=$ Base $\times$ Altura}

No obstante, este triángulo sólo representa el escape de cada fonema. Para poder transformar este valor cualitativo en uno cuantitativo, se calculó el área de un rectángulo, donde uno de sus lados es la base del triángulo y el otro lado la altura gráfica de la pantalla.

Área Rectángulo = Largo x Ancho

Al tener el área del triángulo y el área del rectángulo, se puede determinar el porcentaje de escape de aire nasal de cada fonema estudiado.

Se utilizó la prueba estadística "t" student para determinar la significancia de los resultados obtenidos. 


\section{RESULTADOS}

Al comparar el escape de aire nasal en fonemas oclusivos y fricativos, tal como se ve en el Gráfico 1, se observa un mayor escape de aire en los fonemas fricativos, un 52, 22\%, en cambio, sólo se observa un $35,94 \%$ de escape en fonemas oclusivos, siendo estas diferencias significativas $(p=0.0000)$. Por lo tanto, los fonemas fricativos presentan un mayor escape de aire nasal durante su emisión.

\section{Porcentaje de escape de aire en Fonemas Fricativos v/s Fonemas Oclusivos}

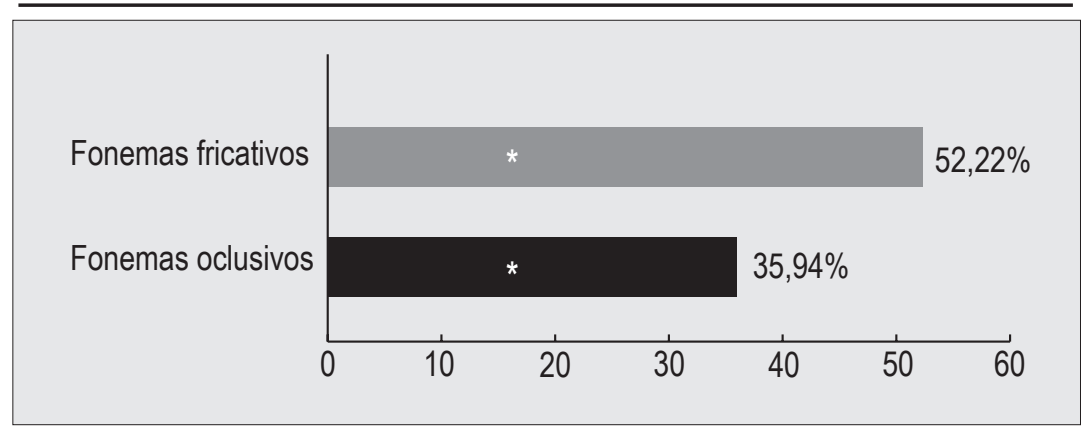

${ }^{*} p=0,0000$

Gráfico 1. Porcentaje de escape de aire nasal en fonemas fricativos y en fonemas oclusivos.

En el Gráfico 2 se observan los porcentajes obtenidos en los fonemas fricativos /s/, /f/ y lj/, encontrándose que el fonema que tiene un mayor porcentaje de escape de aire es el fonema Is/ con un 61,32\%; el fonema fricativo que obtuvo el menor porcentaje fue el fonema lj/ con un 44,94\%; el fonema /f/ obtuvo un $50,41 \%$ de escape de aire nasal. Al comparar dichos fonemas entre sí, se encuentran diferencias significativas en el fonema /s/ $(p=0.0000)$.

Al comparar los fonemas oclusivos entre sí, se encontró un 32,94\% de escape en el fonema $\mid \mathrm{t} /$, un $38,78 \%$ en el fonema $/ \mathrm{p} / \mathrm{y}$ un $36,11 \%$ de escape en el fonema $/ \mathrm{k} /$. Al realizar pruebas estadísticas y compararlos entre sí se encuentran diferencias significativas en los fonemas $/ p / y$ $/ k /(p=0.0000)$, que son los fonemas con mayor grado de escape nasal, tal como se observa en el Gráfico 3. 


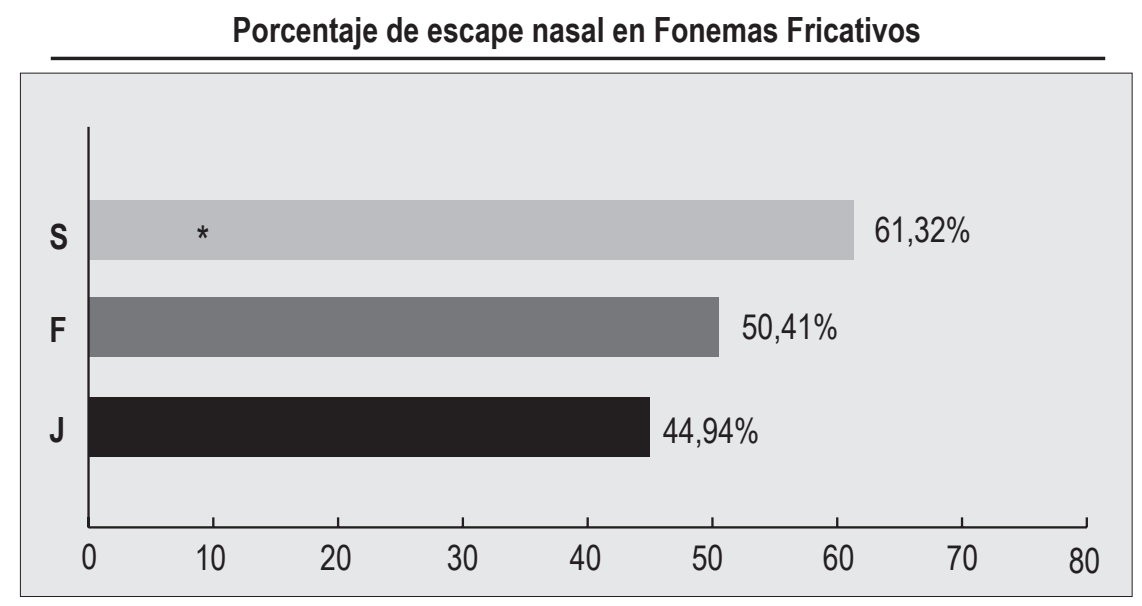

${ }^{*} \mathrm{p}=0,0000$

Gráfico 2. Porcentaje de escape de aire nasal en fonemas fricativos.

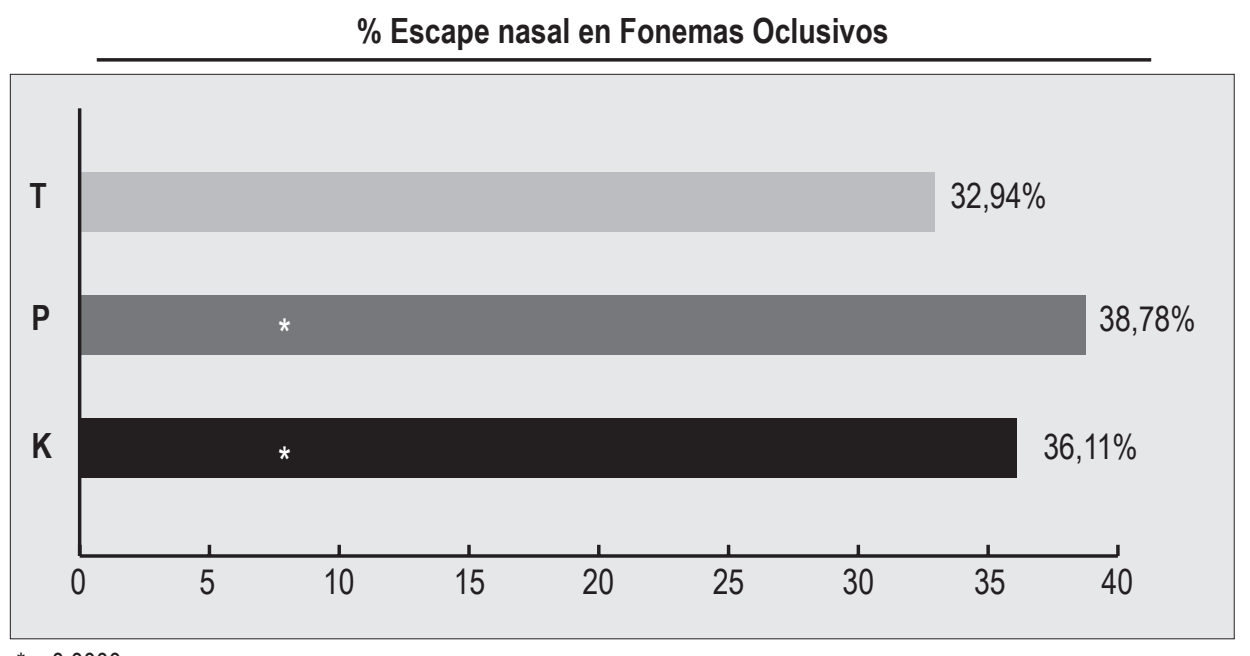

${ }^{*} \mathrm{p}=0,0000$

Gráfico 3. Porcentaje de escape de aire nasal en fonemas oclusivos.

Al comparar el fonema oclusivo más afectado, el fonema /p/, versus el fonema fricativo más alterado, el fonema $/ \mathrm{s} /$, con un $38,78 \%$ y un $61,32 \%$ respectivamente, se encuentran diferencias significativas al realizar pruebas estadísticas ( $p=0.0000)$, tal como se evidencia en el Gráfico 4.

Cuando se comparó el fonema fricativo con menor escape de aire, el fonema lj/ (44.94\%), versus el fonema oclusivo menos afectado, el fonema $/ t /$, con un $32.94 \%$, también se encontraron diferencias significativas ( $p=0.0000$ ), lo que reafirma el mayor porcentaje de escape de aire en los fonemas fricativos que en los fonemas oclusivos, tal como se observa en el gráfico 4. 


\section{Escape nasal en Fonemas}

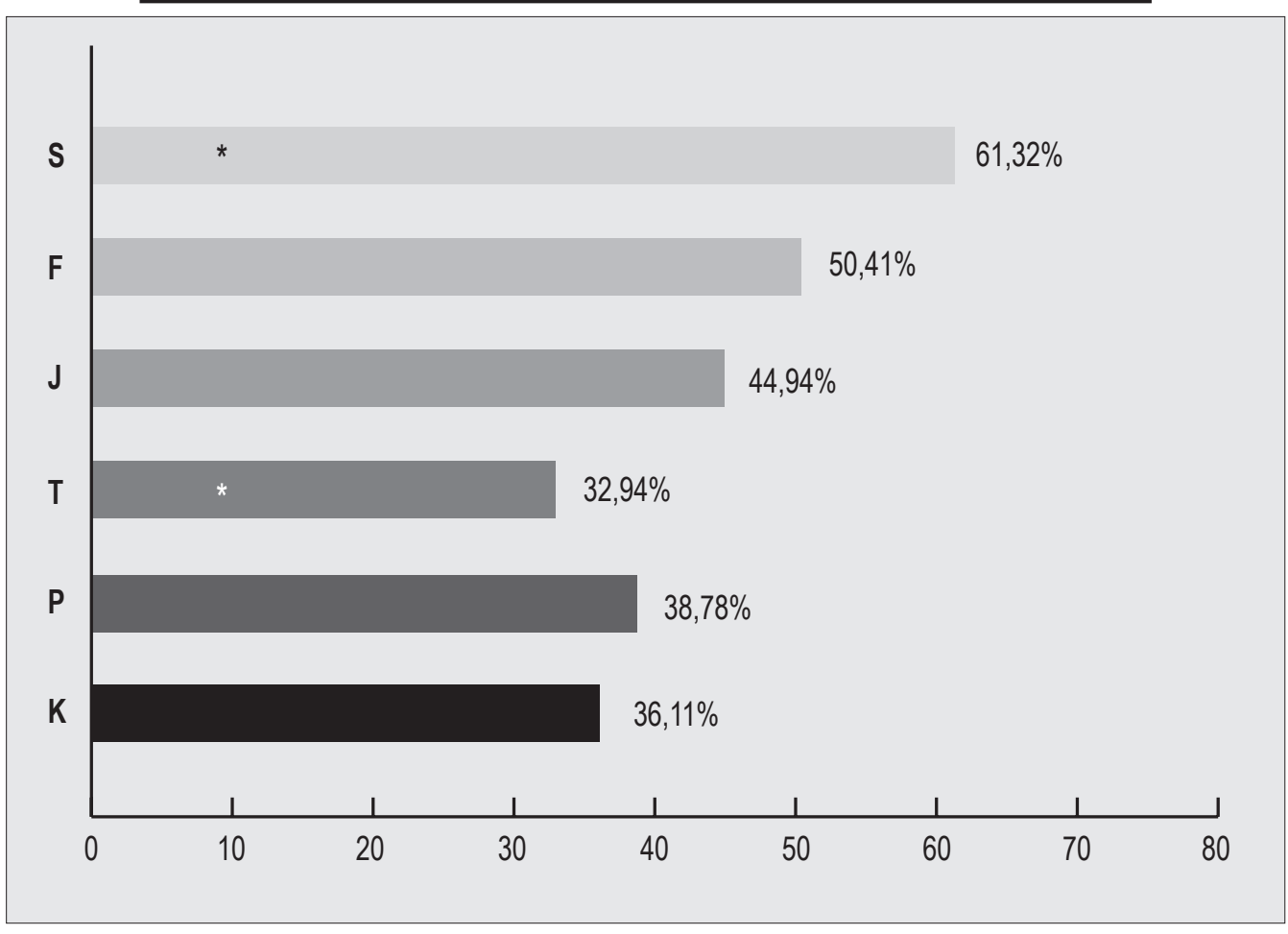

${ }^{*} \mathrm{p}=0,0000$

Gráfico 4. Porcentaje de escape de aire nasal según fonemas.

En la Tabla 1 se observa la cantidad de personas que obtuvieron escapes de aire en los distintos fonemas estudiados. Los fonemas fricativos /s/, /f/ y /j/ fueron los fonemas que más personas tuvieron alterados en comparación con los fonemas oclusivos.

Al comparar la cantidad de fonemas alterados, tanto en los fonemas fricativos y oclusivos, tienden a estar afectados los 3 fonemas, siendo aún mayor la diferencia en los fonemas fricativos donde un $54.05 \%$ de los pacientes evaluados obtuvieron los tres fonemas fricativos alterados, tal como se observa en la Tabla 2.

Tabla 1. Porcentaje de escape de aire por fonemas según cantidad de sujetos afectados.

\begin{tabular}{|c|c|c|c|c|c|c|c|c|c|c|c|}
\hline \multicolumn{2}{|c|}{$|\mathbf{s}|$} & \multicolumn{2}{|c|}{ |fl } & \multicolumn{2}{|c|}{ |j/ } & \multicolumn{2}{|c|}{ It $/$} & \multicolumn{2}{|c|}{$\mid p /$} & \multicolumn{2}{|c|}{$/ \mathrm{k} /$} \\
\hline $\mathrm{n}$ & $\%$ & $\mathrm{n}$ & $\%$ & $\mathrm{n}$ & $\%$ & $n$ & $\%$ & $n$ & $\%$ & $n$ & $\%$ \\
\hline 49 & 66.21 & 50 & 67.56 & 50 & 67.56 & 30 & 40.54 & 39 & 52.70 & 31 & 41.89 \\
\hline
\end{tabular}


Tabla 2. Cantidad de fonemas emitidos

\begin{tabular}{lcrlcr}
\hline & \multicolumn{3}{c}{ Fonemas Fricativos } & \multicolumn{3}{c}{ Fonemas Oclusivos } \\
\hline & $\mathrm{n}$ & $\%$ & & $\mathrm{n}$ & $\%$ \\
1 Fonema & 5 & 6,75 & 1 Fonema & 8 & 10.81 \\
2 Fonemas & 12 & 16,21 & 2 Fonemas & 7 & 9.45 \\
3 Fonemas & 40 & 54,05 & 3 Fonemas & 25 & 33.78 \\
\hline
\end{tabular}

\section{DISCUSIÓN}

Una de las dificultades para poder evaluar a personas portadoras de fisura labiovelopalatinas es la falta de instrumentos objetivamente válidos para poder determinar el escape de aire nasal durante su emisión. Un examen que permite evaluar lo anterior es la llamada aerofonoscopía ${ }^{(13-15)}$.

La aerofonoscopía permite determinar exactamente el escape de aire nasal emitido, lo que proporciona una gran herramienta para determinar el grado de escape de aire nasal que presentan los pacientes portadores de fisuras labiovelopalatinas.

Es sabido que los pacientes con fisura labiovelopalatinas presentan dificultades articulatorias, de lenguaje y emisión de escape de aire nasal ${ }^{(17)}$. Al hablar de emisión de escape de aire nasal, generalmente se menciona que los fonemas más afectados son los fonemas oclusivos, es decir, fonemas $/ \mathrm{p} /$, /t/ y $/ \mathrm{k} /$. Sin embargo, los fonemas que presentan un mayor grado de nasalidad son los fonemas fricativos, según los resultados encontrados en este estudio y lo expuesto por Gómez ${ }^{(3,15)}$ y Bollo(13).

Al comparar los fonemas fricativos versus los fonemas oclusivos estudiados se encontraron diferencias significativas, siendo más afectados los fonemas fricativos $/ \mathrm{s} /$, /f/y lj/, explicable por la falta de movilidad del velo del paladar, por lo tanto, es incompetente el esfinter velofaríngeo. Los fonemas oclusivos están afectados pero en un menor grado y la dificultad de producir dichos fonemas está dada por la baja presión intraoral existente en las personas fisuradas más que en el escape nasal.

Es importante destacar además, que al comparar el fonema fricativo más afectado versus el fonema oclusivo más afectado, también se encontraron diferencias significativas, corroborando que los fonemas fricativos son los más afectados en portadores de fisura labiovelopalatina, lo mismo sucedió al comparar el fonema fricativo menos afectado versus el fonema oclusivo menos afectado.

Un aspecto interesante encontrado fue que al analizar los fonemas fricativos entre sí, se encontraron diferencias significativas sólo en el fonema $/ \mathrm{s} /$. El fonema con un menor escape 
de aire nasal entre los fonemas fricativos resultó ser el fonema /j/. Sería interesante estudiar el por qué de esta situación considerando que el fonema /j/ posee un punto articulatorio velar, implicando un movimiento del velo del paladar.

Asu vez, al estudiar los fonemas oclusivos entre sí, se encontraron diferencias significativas en los fonemas / $\mathrm{p} / \mathrm{y} / \mathrm{k} /$, siendo estos resultados esperables debido a estudios anteriores ${ }^{(16,17)}$.

El desacuerdo en los resultados obtenidos por autores como Henningsson ${ }^{(5)}$, Jones ${ }^{(7)}$, Kummer $^{(8)}$, Lohmander-Agerskov ${ }^{(9)}$, Laczi ${ }^{(16)}$ y Morris ${ }^{(17)}$, se debe a que no contaban con instrumentos que midieran la presión de aire sonora al momento de la emisión de los fonemas, y los resultados de estos autores percibían a los fonemas oclusivos como los más alterados empíricamente.

Gómez ${ }^{(3,15)}$ y Bollo ${ }^{(13)}$ con aerofonoscopía demostraron inequívocamente que la presión de aire al emitir los fonemas fricativos es mayor que la presión de aire al emitir los fonemas oclusivos en pacientes fisurados de paladar y velo.

La explicación más valedera es que los fonemas oclusivos son todos de corta duración en 1000 segundos, mientras que los fonemas fricativos se pueden mantener por mucho más tiempo y, por lo tanto, se posibilita más la pérdida de aire por vía nasal.

Dos explicaciones validan lo expuesto anteriormente:

- El instrumento aerofonoscopio es más preciso.

- El tiempo de duración de los fonemas fricativos es mayor que el de los fonemas oclusivos.

\section{CONCLUSIÓN}

La aerofonoscopía es un examen que permite evaluar objetivamente el escape de aire nasal tanto en personas portadoras o no de fisura labiovelopalatina ${ }^{(13-15)}$. Al ser un examen objetivo permite saber exactamente el escape de aire nasal en palabras o en un fonema determinado.

Con la ayuda de la aerofonoscopía se determinó que los fonemas más afectados son los fonemas fricativos en personas portadoras de fisura labiovelopalatina estudiadas.

El fonema fricativo más afectado fue el fonema /s/. 


\section{REFERENCIAS}

1. Seunghee, H., Hyunsub, S., Minje, Z. \& David., K. (2004) An Acoustic Study of the Temporal Characteristics of Nasalization in Children With and Without Cleft Palate. Cleft Palate Craniofac J, Vol. 41, n5, 535- 543.

2. Álvarez, D., Palomares, M., Quezada, V. \& Villena, C. (2004) Evaluación de la insuficiencia velofaríngea: presentación de un protocolo de evaluación para pacientes portadores de fisura labiopalatina. Revista Chilena de Fonoaudiología, Vol. 5, n², 41-50.

3. Gómez, B. \& Cauvi, D. (2003) Fonoaudiología en el fisurado. En D. Cauvi \& N. Leiva, (Eds.) Etiopatogenia y tratamiento de las fisuras labio-maxilo-palatinas. Santiago, Facultad de Odontología Universidad de Chile. 157-175.

4. Ynsunza, A. \& Pamplona, M. (1992) Diagnóstico y tratamiento de los trastornos de la articulación en el niño con paladar hendido. México: Porrúa.

5. Henningsson, G. \& Isberg A. (1991) Comparison between multiview videofluoroscopy and nasendoscopy of velopharyngeal movements. Cleft Palate Craniofac J, Vol. 28, nº, 413-418.

6. Dalston, R., Warren, D. \& Dalston, E. (1992) A preliminary study of nasal airway patency and its potential effect on speech performance. Cleft Palate Craniofac J, Vol. 29, n4 330-335.

7. Jones, D. (2000) The Relationship Between Temporal Aspects of Oral-Nasal Balance and Classification of Velopharyngeal Status in Speakers with Cleft Palate. Cleft Palate Craniofac J, Vol. 37, n4, 363-369.

8. Kummer, A., Briggs, M. \& Lee, L. (2003) The Relationship Between the Characteristics of Speech and Velopharyngeal Gap Size. Clef Palate Craniofac J, Vol. 40, nº, 590-596.

9. Lohmander-Agerskov, A., Friede, H., Sóderpalm, E. \& Lilja, J. (1997) Residual clefts in the hard palate: correlation between cleft size and speech. Clef Palate Craniofac J, Vol. 34, n², 122-128.

10. Vallino-Napoli, L. \& Montgomery, A. (1997) Examination of standar deviation of mean nasalance scores in subjectswith cleft palate: implications for clinical use. Cleft Palate Craniofac J, Vol. 34, nº, 512- 519.

11. Sánchez, S., García B. \& Regal, N. (1999) Metodología del trabajo logofoniátrico en el paciente fisurado labiopalatino. Revista Cubana de Ortodoncia, Vol. 14, n¹, 7-12.

12. Cortés, J., Niño, A.,Sung, H. \& Gómez B. (2004) Veloplastía funcional secundaria: Una alternativa no obstructiva en el tratamiento de la insuficiencia velofaríngea. Revista Española de Cirugía Oral y Maxilofacial, Vol. 26, $n^{\circ} 2$, 110-120.

13. Bollo, S. (1996) Análisis aerofonoscópico de fonemas vocálicos en pacientes fisurados operados y no operados comparando vocales abiertas y cerradas y determinar el fonema vocálico más nasal. Tesis para optar al Título de Cirujano Dentista. 
14. Ciscutti, V., Calderón, N., Gómez, B. \& Bozan, H. (1997) Aerofonoscopía ventilatoria como alternativa para medir la permeabilidad de las vías aéreas superiores. Magazine International College of Dentists, Vol. 5, $n^{\circ} 1$, 25-30.

15. Gómez, B, Pfister, J. \& Cortés, J. (2000). Duración de fórmulas fonéticas emitidas por pacientes con fisura labio y/o velopalatina operados, medidas mediante el uso de un Aerofonoscopio lic. Revista Odontología Chilena, Vol. 48, n¹, 33-37.

16. Laczi, E., Sussman, J., Stathopoulos, E. \& Huber, J. (2005) Peceptual evaluation of hypernasaltycompared to HONC measures: the role of experience. Clef Palate Craniofac J, Vol. 42, n², 202-211.

17. Morris, H. \& Ozanne, A., (2003) Phonetic, Phonological and Language Skills of Children with cleft palate. Clef Palate Craniofac J, Vol. 40, n5, 460-470.

Recibido : Octubre 2008

Aceptado : : Mayo 2009 